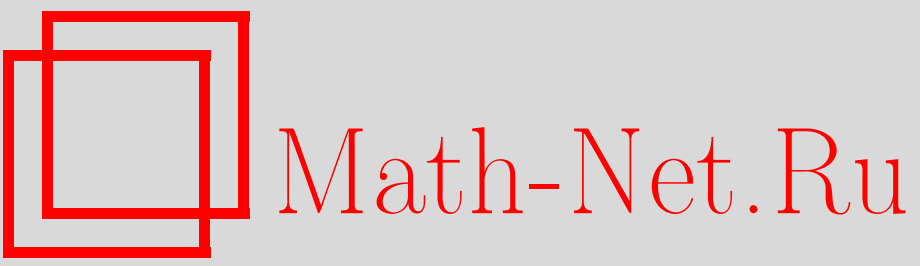

А. Л. Миронов, В. Л. Олейник, О границах применимости метода приближения сильной связи для комплекснозначного потенциала, ТМФ, 1997, том 112 , номер 3, 448-466

DOI: https://doi.org/10.4213/tmf1055

Использование Общероссийского математического портала Math-Net.Ru подразумевает, что вы прочитали и согласны с пользовательским соглашением

http://www.mathnet.ru/rus/agreement

Параметры загрузки:

IP : 35.174 .16 .151

26 апреля 2023 г., 16:15:26 


\author{
ТЕОРЕТИЧЕСКАЯ \\ И МАТЕМАТИЧЕСКАЯ \\ ФИЗИКА \\ Том 112, № 3 \\ сентябрь, 1997
}

А. Л. Миронов* , В. Л. Олейник ${ }^{\dagger}$

\title{
О ГРАНИЦАХ ПРИМЕНИМОСТИ МЕТОДА ПРИБЛИЖЕНИЯ СИЛЬНОЙ СВЯЗИ ДЛЯ КОМПЛЕКСНОЗНАЧНОГО ПОТЕНЦИАЛА
}

\begin{abstract}
Рассматривается одномерный оператор Шредингера с периодическим потенциалом, который строится как сумма сдвигов заданного комплекснозначного потенциала $q$, убывающего на бесконечности. Приведено математическое обоснование метода приближения сильной связи. Пусть $\lambda_{0}$ - изолированное собственное значение оператора Шредингера с потенциалом $q$. Тогда для соответствующего оператора с периодическим потенциалом существует непрерывный спектр, лежащий вблизи $\lambda_{0}$. Изучается асимптотическое поведение этой части спектра при неограниченном возрастании периода для случаев одномерного и двумерного инвариантных подпространств, отвечающих собственному значению $\lambda_{0}$.
\end{abstract}

\section{1. ВВЕДЕНИЕ}

Данная работа является продолжением исследований, начатых авторами в $[1,2]$. Рассматривается одномерный оператор Шредингера с периодическим потенциалом, который строится как сумма сдвигов заданного комплекснозначного одноатомного потенциала $q$ при условии $q \in L^{1}(\mathbf{R})$. Тогда одноатомная задача может иметь дискретньй спектр вне непрерывного [3, 4]. Как известно, спектр периодической цепочки идентичных атомов обладает зонной структурой, т.е. состоит из отрезков вешественной оси для вещественных потенциалов $[5,6]$ и аналитических дуг комплексной плоскости для комплекснозначного потенциала $[7,8,9]$.

Целью наших исследований является получение асимптотических формул для спектральных компонент, ассоциированных с дискретным спектром одноатомной задачи, когда период цепочки неограниченно возрастает. В данном случае часто используется метод приближения сильной связи (tight binding approximation method, tb-метод) [10, $11,12]$. В целях краткости изложения для соответствующих tb-методу явлений и объектов мы будем использовать tb-терминологию (tb-зона, tb-асимптотика и т.п.).

\footnotetext{
* Санкт-Петербургский государственный университет, Санкт-Петербург, Россия. E-mail: mironov@ alm.usr.pu.ru

${ }^{\dagger}$ Санкт-Петербургский государственный университет, Санкт-Петербург, Россия. E-mail: oleinik@ niif.spb.su
} 
Специфика одномерной задачи заключается в том, что рассматриваемый одноатомный оператор не может иметь кратных собственных значений. Тем не менее в случае комплекснозначного потенциала у резольвенты могут существовать кратные полюсы. Рассмотрение комплексного случая в окрестности простого полюса отличается от вешественного незначительно. Известно также, что кратность полюса резольвенты совпадает с размерностью инвариантного подпространства (ИП). Тогда следуя схеме, предложенной в [13], мы получаем, что для периодической задачи вблизи полюса резольвенты одноатомной задачи возникает столько спектральных tb-компонент, какова кратность этого полюса. В настоящей статье мы предлагаем метод исследования асимптотического поведения спектральных компонент, отвечающих соответствуюшему собственному значению $\lambda_{0}$ одноатомной задачи для ИП размерности 1 и 2 .

Полученные результаты показывают, что поведение спектральных компонент может быть весьма разнообразным. Наиболее простая ситуация возникает, когда у нас имеется одномерное ИП. В этом случае асимптотически спектр в окрестности соответствующего собственного значения $\lambda_{0}$ состоит из отрезка и при увеличении периода этот отрезок, врашаясь вокруг своего центра, экспоненциально сжимается в точку $\lambda_{0}$. Далее в статье рассмотрен случай ИП размерности 2 , т.е. собственному значению $\lambda_{0}$, помимо собственной функции, отвечает одна присоединенная. Здесь выявляются два качественно различных варианта спектральной картины вблизи $\lambda_{0}$. В первом варианте, когда потенциал убывает медленно, мы наблюдаем две спектральные компоненты, асимптотически представляющие собой два симметричных относительно $\lambda_{0}$ отрезка, каждый из которых ведет себя аналогично предыдущему случаю. Однако если потенциал убывает достаточно быстро или вообше финитен, то спектральная картина может выродиться в "крест", что не противоречит общей теории спектральных свойств уравнения Хилла $[9,14,15]$. При этом точка смыкания tb-зон, образуюших “крест”, возможна лишш при одинаковом для каждой из зон значении квазиимпульса, поскольку эта точка не может быть устойчивой [16].

В разделе 1 рассматривается одноатомная задача и выписываются асимптотические решения этой задачи. Соответствуюшая периодическая задача рассматривается в разделе 2 и там же приводятся уравнения, описьвающие зонную структуру спектра в терминах решений периодической задачи. Эти решения строятся в разделе 3 из решений одноатомной задачи методом вариации произвольных постоянных. После этого в разделе 4 получается основное уравнение (4.2), которое в удобных для нас терминах описывает зону, соответствующую фиксированному собственному значению одноатомной задачи $\lambda_{0}$ (tb-зону). В дальнейшем мы занимаемся решением уравнения (4.2). В том же разделе выводятся условия одномерности и двумерности ИП, отвечаюшего $\lambda_{0}$. Далее, в разделах 5 и 6 рассматривается случай одномерного ИП и как главный результат приводится теорема 2. Случай же двумерного ИП рассмотрен в разделах 7 и 8 , а основной результат выражается теоремой 4. В последнем разделе даны конкретные примеры, которые допускают как непосредственное вычисление tb-асимптотик, так и их расчет с использованием теорем 2 и 4.

4 Теоретическая и математическая физика, т. 112, № 3, 1997 г 


\section{1. ОДИн АТОМ}

Рассмотрим в $L^{2}(\mathbf{R})$ уравнение Шредингера с комплекснозначным абсолютно суммируемым потенциалом $q(x)$ :

$$
\begin{gathered}
-y^{\prime \prime}(x)+q(x) y(x)=\lambda y(x), \quad x \in \mathbf{R}, \\
\int_{-\infty}^{+\infty}|q(x)| d x<\infty .
\end{gathered}
$$

Спектр такой задачи $\sigma \subset \mathbf{C}$ состоит из вешественной полуоси $[0,+\infty)$ и некоторого не более чем счетного числа собственных значений, лежаших в комплексной плоскости вне этой полуоси [17].

Для одного из таких собственных значений $\lambda_{0} \notin[0,+\infty)$ выбираем два решения $\theta(x)$ и $\varphi(x)$ уравнения (1.1) при $\lambda=\lambda_{0}$, первое из которых есть нормированная в $L^{2}(\mathbf{R})$ собственная функция, а второе выбирается так, чтобы определитель Вронского этих решений $W[\theta, \varphi]$ равнялся 1 . Положим $\rho:=\sqrt{\lambda_{0}}, \eta:=\operatorname{Im} \rho>0$.

Для выбранных решений сушествуют положительные постоянные $C_{\theta}$ и $C_{\varphi}$ такие, что

$$
|\theta(x)| \leq C_{\theta} e^{-\eta|x|}, \quad|\varphi(x)| \leq C_{\varphi} e^{\eta|x|}, \quad-\infty<x<+\infty .
$$

При дальнейших оценках нам удобно использовать следуюшую функцию:

$$
p(T):=\int_{|x| \geq T}|q(x)| d x+e^{-2 \eta T}, \quad T>0 .
$$

Функция $p(T)$ монотонно стремится к нулю при $T \rightarrow \infty$, и аналогично лемме 1 из [2] имеем:

Лемма 1. Существуют постоянные $C_{ \pm} \neq 0$ такие, что при $x \rightarrow \pm \infty$

$$
\begin{aligned}
\theta(x) & =C_{ \pm} e^{i \rho|x|}(1+O(p(|x|))), & \varphi(x) & =\mp\left(2 i \rho C_{ \pm}\right)^{-1} e^{-i \rho|x|}(1+O(p(|x| / 2))), \\
\theta^{\prime}(x) & = \pm i \rho C_{ \pm} e^{i \rho|x|}(1+O(p(|x|))), & \varphi^{\prime}(x) & =\left(2 C_{ \pm}\right)^{-1} e^{-i \rho|x|}(1+O(p(|x| / 2))) .
\end{aligned}
$$

Положим $[f, g](x):=f(x) g^{\prime}(-x)-f^{\prime}(x) g(-x)$. Тогда для выбранных решений $\theta(x)$ и $\varphi(x)$ уравнения (1.1) как следствие леммы 1 при $x \rightarrow \pm \infty$ получаем следующие асимптотические формулы:

$$
\begin{gathered}
a(x):=[\varphi, \varphi](x)=\mp\left(2 i \rho C_{+} C_{-}\right)^{-1} e^{-2 i \rho|x|}(1+O(p(|x| / 2))), \\
b(x):=[\theta, \varphi](x)=O(p(|x| / 2)), \\
c(x):=[\theta, \theta](x)=\mp 2 i \rho C_{+} C_{-} e^{2 i \rho|x|}(1+O(p(|x|))) .
\end{gathered}
$$




\section{2. ПЕРИОДИЧЕСКАЯ ЦЕПОЧКА ИДЕНТИЧНЫХ АТОМОВ}

Для некоторого фиксированного $T>0$, используя потенциал $q(x)$, построим периодический потенциал $Q(x ; T)$ (кратко $Q(x)$ или $Q$ ) с периодом $2 T$ :

$$
Q(x ; T):=\sum_{n=-\infty}^{+\infty} q(x+2 T n) .
$$

В этом определении сходимость ряда в $L^{1}$ по любому конечному промежутку гарантируется условием (1.2).

Рассмотрим теперь уравнение Шредингера

$$
-y^{\prime \prime}(x)+Q(x ; T) y(x)=\lambda y(x), \quad x \in \mathbf{R} .
$$

Спектр $\sigma(Q)$ этой цепочки состоит не более чем из счетного числа криволинейных отрезков (спектральных компонент), гомеоморфных единичному отрезку $[0,1]$ и расположенных в комплексной плоскости [7-9]. Эти криволинейные отрезки могут быть описаны следующим образом [6]:

$$
\sigma(Q)=\{\lambda \in \mathbf{C}: F(\lambda, T) \in[-2,2]\},
$$

где $F(\lambda, T)$ - дискриминант уравнения (2.2). В действительности это означает вьполнение следуюших двух вещественных условий:

$$
\left\{\begin{array}{l}
|\operatorname{Re} F(\lambda, T)| \leq 2 \\
\operatorname{Im} F(\lambda, T)=0
\end{array}\right.
$$

В этой системе второе условие определяет семейство кривых на комплексной плоскости, а первое условие выделяет на этих кривых искомые спектральные компоненты. При этом границы спектральных компонент определяются формулой

$$
F(\lambda, T)= \pm 2 .
$$

Пусть $\theta_{T}$ и $\varphi_{T}$ - два линейно независимых решения уравнения $(2.2)$ с $W\left[\theta_{T}, \varphi_{T}\right] \equiv 1$, тогда (ср. [18])

$$
F(\lambda, T)=\left[\theta_{T}, \varphi_{T}\right](T)+\left[\theta_{T}, \varphi_{T}\right](-T) .
$$

Выразив функции $\varphi_{T}(x)$ и $\theta_{T}(x)$ в удобных для нас терминах и подставив эти выражения в (2.4), мы придем к основному уравнению.

\section{3. ПОСТРОЕНИЕ ФУНКЦИЙ $\varphi_{T}(x)$ И $\theta_{T}(x)$}

Построение функций $\varphi_{T}(x)$ и $\theta_{T}(x)$ идентично построению соответствующих функций, проведенному в разделе 3 работы [2]. Тем не менее для удобства читателей мы кратко повторяем его. 
Исходя из выбранных в разделе 2 функций $\varphi$ и $\theta$ методом вариации произвольных постоянных построим решения $\varphi_{T}(x)$ и $\theta_{T}(x)$. Если положить

$$
\kappa=\lambda-\lambda_{0}, \quad v(x, T)=Q(x, T)-q(x), \quad w(x, \kappa, T)=v(x, T)-\kappa,
$$

то уравнение Шредингера (2.2) запишется в виде

$$
-y^{\prime \prime}(x)+\left(q(x)-\lambda_{0}\right) y(x)=-w(x, \kappa, T) y(x) .
$$

При этом заметим, что функции $\varphi(x)$ и $\theta(x)$ являются решениями уравнения $(3.2)$ с правой частью, равной нулю. Пусть

$$
\varphi_{T}(x)=\alpha(x)(\varphi(x)+\gamma(x) \theta(x))
$$

где $\alpha(x)$ и $\gamma(x)$ удовлетворяют условиям

$$
\alpha(0)=1, \quad \gamma(0)=0, \quad \alpha^{\prime}(x) \varphi(x)+(\alpha(x) \gamma(x))^{\prime} \theta(x)=0
$$

Из (3.3) и (3.4) следует, что $\varphi_{T}(0)=\varphi(0), \varphi_{T}^{\prime}(0)=\varphi^{\prime}(0)$. Тогда функция $\varphi_{T}(x)$ будет решением уравнения $(3.2)$, если функции $\alpha(x)$ и $\gamma(x)$ являются решениями следуюшей системы нелинейных интегральных уравнений Вольтерра:

$$
\begin{aligned}
& \alpha(x)=\exp \int_{0}^{x} w \theta(\varphi+\gamma \theta) d \xi \\
& \gamma(x)=-\int_{0}^{x} w(\varphi+\gamma \theta)^{2} d \xi .
\end{aligned}
$$

При выводе (3.5), (3.6) мы воспользовались соотношениями (3.4) и условием $W[\theta, \varphi]=1$. Как будет показано ниже, система (3.5), (3.6) однозначно разрешима в подходяшем пространстве.

Далее, построим решение $\theta_{T}(x)$ уравнения (3.2) так, чтобы $W\left[\theta_{T}, \varphi_{T}\right] \equiv 1$. Для этого положим

$$
\theta_{T}(x)=\alpha^{-1}(x) \theta(x)+\beta(x) \varphi_{T}(x)
$$

где функция $\beta(x)$ такая, что $\beta(0)=0$ и

$$
\left(\alpha^{-1}(x)\right)^{\prime} \theta(x)+\beta^{\prime}(x) \varphi_{T}(x)=0 .
$$

Из (3.7) и (3.8) мы имеем $\theta_{T}^{\prime}=\alpha^{-1} \theta^{\prime}+\beta \varphi_{T}^{\prime}$ и, т.к. $\theta(x) \varphi_{T}^{\prime}(x)-\theta^{\prime}(x) \varphi_{T}(x)=\alpha(x)$, то вронскиан функций $\theta_{T}$ и $\varphi_{T}$ равен 1 . Кроме того, $\theta_{T}(0)=\theta(0), \theta_{T}^{\prime}(0)=\theta^{\prime}(0)$ и функция $\theta_{T}(x)$ является решением уравнения (3.2) при

$$
\beta(x)=\int_{0}^{x} w \theta^{2} \alpha^{-2} d \xi
$$


Заметим, что функции $\alpha, \gamma$ и $\beta$ зависят от $x, \kappa, T$ и $\lambda_{0}=\rho^{2}$.

\section{4. ОСНОВНОЕ УРАВНЕНИЕ}

Подставляя выражения $(3.3)$ для $\varphi_{T}(x)$ и $(3.7)$ для $\theta_{T}(x)$ в выражение $(2.4)$ и используя обозначения (1.5)-(1.7), мы приходим к равенству

$$
\begin{aligned}
F\left(\kappa+\lambda_{0}, T\right)=[ & \beta(T)-\beta(-T)] \alpha(T) \alpha(-T)\{a(T)+\gamma(T) b(T)- \\
& -\gamma(-T) b(-T)+\gamma(T) \gamma(-T) c(T)\}+ \\
& +\{(b(T)+c(T) \gamma(-T)) \alpha(-T) / \alpha(T)+ \\
& +(b(-T)+c(-T) \gamma(T)) \alpha(T) / \alpha(-T)\} .
\end{aligned}
$$

Используя сокрашенные обозначения

$G:=\alpha_{-} \alpha_{+}\left(a+\gamma_{+} b_{+}+\gamma_{-} b_{-}+\gamma_{-} \gamma_{+} c\right), \quad H:=\left(b_{+}+\gamma_{-} c\right) \alpha_{-} / \alpha_{+}-\left(b_{-}+\gamma_{+} c\right) \alpha_{+} / \alpha_{-}$, где $\alpha_{ \pm}:=\alpha( \pm T) ; \gamma_{ \pm}:=\gamma( \pm T) ; a:=a(T) ; b_{ \pm}:= \pm b( \pm T)$ и $c:=c(T)=-c(-T), \mathrm{c}$ учетом (3.9), равенство (4.1) можно переписать в виде

$$
F\left(\kappa+\lambda_{0} ; T\right)=G \int_{-T}^{T}(v-\kappa) \theta^{2} \alpha^{-2} d \xi+H .
$$

Заметим, что функции $G$ и $H$ зависят от $\kappa, T$ и $\rho$. Согласно (2.3) для каждого $d \in[-2,2]$ уравнение $F(\lambda, T)=d$ определяет точку спектра $\lambda(d ; T) \in \sigma(Q)$. Запишем это уравнение следуюшим образом:

$$
\kappa\left(\int_{-T}^{T} \theta^{2} \alpha^{-2} d x\right)=\int_{-T}^{T} v \theta^{2} \alpha^{-2} d x+(H-d) G^{-1} .
$$

В дальнейшем мы изучим асимптотическое поведение того решения $\kappa(d ; T)=$ $\lambda(d ; T)-\lambda_{0}$ уравнения (4.2), которое стремится к нулю при $T \rightarrow+\infty$. Здесь необходимо отметить, что интеграл в левой части равенства (4.2) может стремиться к нулю. В этом формально проявляется специфика несамосопряженности задачи, рассматриваемой для комплекснозначного потенциала. Как будет показано ниже, характер асимптотического поведения вышеуказанного интеграла непосредственно связан с размерностью ИП, отвечающего собственному значению $\lambda_{0}$ одноатомной задачи. В силу нормировки собственной функции $\theta$ этот интеграл стремится к 1 для вещественного потенциала [2]. Ниже мы рассмотрим случаи одномерного и двумерного ИП. Для этого выразим в терминах функций $\theta$ и $\varphi$ условия, при которых размерность ИП, отвечаюшего собственному значению $\lambda_{0}$, равна 1 либо 2 .

ЛЕмма 2. Пусть

$$
\mathcal{I}_{1}:=\int_{-\infty}^{+\infty} \theta^{2}(x) d x, \quad \mathcal{I}_{2}:=2 \int_{-\infty}^{+\infty} \theta^{2}(x)\left(\int_{0}^{x} \theta\left(x_{1}\right) \varphi\left(x_{1}\right) d x_{1}\right) d x .
$$

Тогда:

а) для того чтобь ИП, отвечающее собственному значению $\lambda_{0}$, бълло одномерныцм, необходимо и достаточно, чтобь $\mathcal{I}_{1} \neq 0$;

б) для того чтобъ ИП, отвечающее собственному значению $\lambda_{0}$, бъло двумерныцм, необходимо и достаточно, чтобы $\mathcal{I}_{1}=0$ и $\mathcal{I}_{2} \neq 0$. 
ДокАЗАТЕльСтво: а) некоторая функция $y(x)$ есть первая присоединенная функция, отвечающая собственному значению $\lambda_{0}$, если она удовлетворяет уравнению

$$
-y^{\prime \prime}(x)+q(x) y(x)-\lambda_{0} y(x)=\theta(x) .
$$

Условием разрешимости этого уравнения в $L^{2}(\mathbf{R})$ является ортогональность правой части к решению сопряженного однородного уравнения (4.4), т.е.

$$
\int_{-\infty}^{+\infty} \theta^{2}(x) d x=0
$$

если же это условие нарушается, то ИП одномерно;

б) допустим, что ИП двумерно, тогда присоединенный вектор $\theta_{1}$, отвечающий $\lambda_{0}$, запишется в виде

$$
\theta_{1}(x)=\theta(x) \int_{0}^{x} \theta\left(x_{1}\right) \varphi\left(x_{1}\right) d x_{1}-\varphi(x) \int_{-\infty}^{x} \theta^{2}\left(x_{1}\right) d x_{1} .
$$

Следовательно, сушествование еще одного корневого вектора равносильно разрешимости уравнения (4.4) с функцией $\theta_{1}$ в правой части, что, в свою очередь, равносильно ортогональности функций $\theta_{1}$ и $\bar{\theta}$. Нарушение условия ортогональности приводит к условиям $\mathcal{I}_{1}=0$ и $\mathcal{I}_{2} \neq 0$. Лемма доказана.

\section{5. ВСПОМОГАТЕЛЬНЫЕ ОЦЕНКИ В СЛУЧАЕ ОДНОМЕРНОГО ИНВАРИАНТНОГО ПОДПРОСТРАНСТВА}

Так же как в [2], наряду с функцией $p(T)$, определяемой формулой $(1.4)$, введем в рассмотрение еще одну оценочную функцию

$$
r_{1}(T):=\sup _{t \geq T} \int_{-t}^{t}|v(x, t)| e^{-2 \eta|x|} d x+e^{-2 \eta T}, \quad T>0 .
$$

Для $T>0$ имеем $r_{1}(T) \leq p(T)$. Примеры поведения функций $p(T)$ и $r_{1}(T)$ для различных потенциалов $q(x)$ сходны с примерами, рассмотренными в [2].

Далее, для некоторого произвольного фиксированного положительного числа $C_{\kappa}$ и $T>0$, ограничивая область исследования спектра $\sigma(Q)$ на окрестность точки $\lambda_{0}$, мы предполагаем, что

$$
|\kappa| \leq C_{\kappa} r_{1}(T)
$$

Предположение (5.2) выделяет на комплексной плоскости круг $B_{1}(T)$ радиуса $C_{\kappa} r_{1}(T)$ с центром в нуле, на котором рассматривается уравнение (4.2) и проводятся все вспомогательные оценки.

ЛЕмма 3. Для произвольного числа $C_{\kappa}>0$ существует положительное число $T_{0}$ такое, что для любого $T \geq T_{0}$ и любого $\kappa \in B_{1}(T)$ уравнение (3.6) разрешимо в $C(\mathbf{R})$. Решение $\gamma(x)=\gamma(x, \kappa)$ единственно, и при $|x| \leq T$ справедливы оценки

$$
\begin{array}{rlrlrl}
|\gamma(x)| & \leq C_{\gamma} p(T) e^{2 \eta|x|} & & u & & \left|\partial_{\kappa} \gamma(x)\right| \leq C_{\gamma}^{\prime} e^{2 \eta|x|}, \\
|\ln \alpha(x)| & \leq C_{\alpha}\left(|x| r_{1}(T)+p(T)\right) & & u & \left|\partial_{\kappa} \ln \alpha(x)\right| \leq C_{\alpha}^{\prime}|x|,
\end{array}
$$


где $C_{\gamma}, C_{\gamma}^{\prime}, C_{\alpha}$ и $C_{\alpha}^{\prime}$ - некоторые постоянные. Можно положить

$$
\begin{array}{lll}
C_{\gamma}=\left(1+C_{\kappa} / \eta\right)\left(C_{\varphi}+C_{\theta}\right)^{2} & u & C_{\gamma}^{\prime}=2\left(C_{\varphi}+C_{\theta}\right)^{2} / \eta, \\
C_{\alpha}=C_{\varphi} C_{\theta}\left(C_{\kappa}+1\right) & u & C_{\alpha}^{\prime}=C_{\varphi} C_{\theta}+1 .
\end{array}
$$

Лемма 3 является полным аналогом соответствующих лемм из $[1,2]$ и доказывается теми же средствами. Так же аналогично соответствуюшим леммам из $[1,2]$ доказываются следуюшие ниже леммы 4-6.

Лемма 4. При $T \rightarrow+\infty$ имеют место равномерные относительно $\kappa \in B_{1}(T)$ и $x \in[-T, T]$ асимптотические формуль

$$
\begin{aligned}
\gamma(x) & =\int_{0}^{x}(\kappa-v(\xi, T)) \varphi^{2}(\xi) d \xi+e^{2 \rho|x|} O\left(p^{2}(T)\right), \\
\partial_{\kappa} \gamma(x) & =\int_{0}^{x} \varphi^{2}(\xi) d \xi+e^{2 \rho|x|} O(p(T)), \\
\ln \alpha(x) & =-\kappa \int_{0}^{x} \theta(\xi) \varphi(\xi) d \xi+O\left(p(T)\left(1+|x| r_{1}(T)\right)\right), \\
\partial_{\kappa} \ln \alpha(x) & =-\int_{0}^{x} \theta(\xi) \varphi(\xi) d \xi+|x| O(p(T)) .
\end{aligned}
$$

Лемма 5. Пусть $C_{\kappa}, C_{\alpha}, C_{\alpha}^{\prime}, C_{g}$ и $C_{g}^{\prime}$ - положительные числа. Причем $C_{\alpha}$ и $C_{\alpha}^{\prime}$ такие же, как в лемме $3, a C_{g}$ и $C_{g}^{\prime}$ удовлетворяют условиям $C_{g}>2\left|\rho C_{+} C_{-}\right|$ $u C_{g}^{\prime}>C_{\alpha}^{\prime}\left|\rho C_{+} C_{-}\right|^{-1}$, mогда:

а) существуют положительнье числа $T_{1}, C_{h}$ и $C_{h}^{\prime}$ такие, что для любого $T \geq T_{1}$ и любого $\kappa \in B_{1}(T)$ справедливы неравенства

$$
\begin{aligned}
|G(\kappa, T)|^{-1} & \leq C_{g} \exp \left[-2 \eta T+2 C_{\alpha} T r_{1}(T)\right], \\
\left|\partial_{\kappa} G(\kappa, T)\right| & \leq C_{g}^{\prime} T \exp \left[2 \eta T+2 C_{\alpha} \operatorname{Tr}_{1}(T)\right], \\
|H(\kappa, T)| & \leq C_{h} p(T / 2) \exp \left[2 C_{\alpha} \operatorname{Tr}_{1}(T)\right], \\
\left|\partial_{\kappa} H(\kappa, T)\right| & \leq C_{h}^{\prime}(1+T p(T / 2)) \exp \left[2 C_{\alpha} T r_{1}(T)\right] ;
\end{aligned}
$$

б) равномерно относительно $\kappa \in B_{1}(T)$ при $T \rightarrow+\infty$ имеет место асимптотическая формула

$$
\begin{aligned}
& G^{-1}(\kappa, T)=- 2 i \rho C_{+} C_{-}(1+O(p(T / 2))) \times \\
& \times \exp \left[2 i \rho T+\kappa\left(\int_{0}^{T}-\int_{-T}^{0}\right) \theta(x) \varphi(x) d x+O\left(T p(T) r_{1}(T)\right)\right] .
\end{aligned}
$$

ЛЕмма 6. При $T \rightarrow+\infty$

$$
\begin{aligned}
\int_{-T}^{T} \theta^{2}(x) \alpha^{-2}(x) d x & =\mathcal{I}_{1}+O(p(T)) \\
\int_{-T}^{T} v(x) \theta^{2}(x) \alpha^{-2}(x) d x & =\int_{-T}^{T} v(x) \theta^{2}(x) d x+O\left(p(T) r_{1}(T)\right)
\end{aligned}
$$

равномерно относительно $\kappa \in B_{1}(T)$. 


\section{6. СУЩЕСТВОВАНИЕ И АСИМПТОТИЧЕСКОЕ ПОВЕДЕНИЕ СПЕКТРАЛЬНОЙ КОМПОНЕНТЫ В СЛУЧАЕ ОДНОМЕРНОГО ИНВАРИАНТНОГО ПОДПРОСТРАНСТВА}

Верна следующая теорема.

ТЕОрема 1. Пусть $\lambda_{0}-$ собственное значение одноатомной задачи (1.1) с одномерным ИП, т.е. $\mathcal{I}_{1} \neq 0$. Пусть $C_{\kappa}$ и $d_{0}$ - положсительные числа, удовлетворяющие условиям $C_{\kappa}>\left|\mathcal{I}_{1}\right|^{-1} \max \left\{C_{\theta}^{2}, 2\left|\rho C_{+} C_{-}\right| d_{0}\right\}$, если $\operatorname{Tr}_{1}(T) \rightarrow 0$ при $T \rightarrow+\infty, u$ $C_{\kappa}>\left|\mathcal{I}_{1}\right|^{-1} C_{\theta}^{2}$ в противном случае. Тогда существует положсительное число $t_{0}$ такое, что для любого $T \geq t_{0}$ и любого $d \in\left[-d_{0}, d_{0}\right]$ уравнение $(4.2)$ в круге $B_{1}(T)$ имеет одно и только одно решение $\kappa=\kappa(d ; T)$.

ДокАЗАтЕльство. Воспользуемся теоремой Руше для круга $B_{1}(T)$. Запишем уравнение (4.2) в виде $\kappa=f(\kappa)$, где $f(\kappa)$ - аналитическая в круге функция, зависящая также от $d, T$ и $\lambda_{0}$ :

$$
f(\kappa)=\left[\int_{-T}^{T} v \theta^{2} \alpha^{-2} d x+(H-d) G^{-1}\right]\left(\int_{-T}^{T} \theta^{2} \alpha^{-2} d x\right)^{-1} .
$$

Используя результаты предыдушего раздела, оценим модуль функции $f(\kappa)$ на границе круга $B_{1}(T)$ при $T \rightarrow+\infty$ :

$$
\begin{aligned}
|f(\kappa)| \leq & {\left[\int_{-T}^{T}|v||\theta|^{2} d x(1+o(1))+\left(d_{0}+|H|\right) /|G|\right]\left(\left|\mathcal{I}_{1}\right|^{-1}+o(1)\right) \leq } \\
\leq & \left|\mathcal{I}_{1}\right|^{-1} \int_{-T}^{T}|v||\theta|^{2} d x+o\left(r_{1}(T)\right)+\left(d_{0}+C_{h} p(T / 2) \exp \left[2 C_{\alpha} \operatorname{Tr}_{1}(T)\right]\right) \times \\
& \times 2\left|\rho C_{+} C_{-}\right|\left|\mathcal{I}_{1}\right|^{-1}(1+o(1)) \exp \left[-2 \eta T+2 C_{\alpha} \operatorname{Tr}_{1}(T)\right] .
\end{aligned}
$$

В случае $\operatorname{Tr}_{1}(T) \rightarrow 0$ при $T \rightarrow+\infty$ для больших $T$ имеем

$$
|f(\kappa)| \leq\left|\mathcal{I}_{1}\right|^{-1} \int_{-T}^{T}|v||\theta|^{2} d x+2\left|\rho C_{+} C_{-}\right|\left|\mathcal{I}_{1}\right|^{-1} d_{0} e^{-2 \eta T}+o\left(r_{1}(T)\right)<C_{\kappa} r_{1}(T) .
$$

Если $\operatorname{Tr}_{1}(T)$ не стремится к нулю, то для всех достаточно больших $T$ сушествует такое $t_{0}$, что при $T \geq t_{0}$ выполняется неравенство $-2 \eta+4 C_{\alpha} r_{1}(T)<-\eta$. А т.к. $e^{-\eta T}=o\left(r_{1}(T)\right)$, то мы получаем

$$
|f(\kappa)| \leq\left|\mathcal{I}_{1}\right|^{-1} \int_{-T}^{T}|v||\theta|^{2} d x+o\left(r_{1}(T)\right) \leq\left|\mathcal{I}_{1}\right|^{-1} C_{\theta}^{2} r_{1}(T)(1+o(1))<C_{\kappa} r_{1}(T)
$$

Следовательно, сушествует $t_{0}>0$ такое, что при $T \geq t_{0}$ на границе круга $B_{1}(T)$ верно неравенство $|f(\kappa)|<|\kappa|$. Тогда согласно теореме Руше функция $f(\kappa)-\kappa$ имеет внутри круга $B_{1}(T)$ столько же нулей, сколько функция $\kappa$, т.е. ровно один. Теорема доказана.

В соответствии с формулой (2.3) имеем следуюшее следствие теоремы 1. 
СлеДСтвиЕ 1. Пусть $d_{0} \geq 2, \quad \mathcal{I}_{1} \neq 0, C>\left|\mathcal{I}_{1}\right|^{-1} \max \left\{C_{\theta}^{2}, 2\left|\rho C_{+} C_{-}\right| d_{0}\right\}$ и пусть $\kappa(d ; T)$ при $|d| \leq d_{0}$ является таким решением уравнения (4.2), что $|\kappa(d ; T)| \leq$ $C r_{1}(T)$ для больших T. Положим согласно $(3.1) \lambda(d ; T)=\lambda_{0}+\kappa(d ; T)$. Тогда, для того чтобь $\lambda(d ; T) \in \sigma(Q)$, необходимо и достаточно, чтобы $d \in[-2,2]$. Следовательно, криволинейный отрезок $\{\lambda(d ; T):-2 \leq d \leq 2\}$ совпадает с изолированной для больших $T$ спектральной компонентой, лежащей вблизи $\lambda_{0}$.

Таким образом мы доказали сушествование спектральной компоненты вблизи $\lambda_{0}$. Приведем теперь формулы, асимптотически описьвающие эту компоненту.

Tеорема 2. Пусть функиия $q(x) \in L^{1}(\mathbf{R})$, число $T>0$ и функиия $Q(x ; T)$ определена формулой (2.1). Пусть $\lambda_{0} \notin[0,+\infty)$ - собственное значение и $\theta(x) \in L^{2}(\mathbf{R})$ соответствующая собственная функиия задачи (1.1) такая, что $\|\theta\|_{L^{2}(R)}=1 u$ $\mathcal{I}_{1}:=\int_{-\infty}^{+\infty} \theta^{2}(x) d x \neq 0$. Пусть функиия $\varphi(x)-$ какое-либо решение уравнения (1.1) при $\lambda=\lambda_{0}$ такое, что $W[\theta, \varphi] \equiv 1$. Положим $V(T):=\int_{-T}^{T}(Q(x ; T)-q(x)) \theta^{2}(x) d x$, $U(T):=\left(\int_{0}^{T}-\int_{-T}^{0}\right) \theta(x) \varphi(x) d x$. Пусть, далее, $\rho=\sqrt{\lambda_{0}}, \operatorname{Im} \rho>0$, иисла $C_{\theta}$ и $C_{ \pm}$ такие же, как в (1.3) и лемме 1, а функиии $p(T)$ и $r_{1}(T)$ определены формулами (1.4) и (5.1), соответственно. Тогда, если $C>\left|\mathcal{I}_{1}\right|^{-1} \max \left\{C_{\theta}^{2}, 4\left|\rho C_{+} C_{-}\right|\right\}$и $\kappa(d ; T)$ - решение уравнения (4.2) при $d \in[-2,2]$ такое, что $|\kappa(d ; T)| \leq C r_{1}(T)$ для больших $T$, то верны следующие утверждения:

1) $\lambda(d ; T)=\lambda_{0}+\kappa(d ; T) \in \sigma(Q)$ nрu $d \in[-2,2]$;

2) $\lambda( \pm 2 ; T)$ - края спектральной компоненты;

3) имеет место асимптотическая формула

$$
\lambda(0 ; T)=\lambda_{0}+\left[V(T) / \mathcal{I}_{1}\right]+\varepsilon_{1}(T),
$$

əде $\varepsilon_{1}(T)=o\left(r_{1}(T)\right)$ при $T \rightarrow+\infty$;

4) справедлива формула для параметрического представления спектральной компоненты

$$
\lambda(d ; T)=\lambda(0 ; T)+\Delta(d ; T) d e^{2 i \rho T},
$$

причем для $\Delta(d ; T)$ верны следующие асимптотические формуль:

а) в общем случае

$$
\ln [\Delta(d ; T)]=V(T) U(T)+\varepsilon_{2}(T)
$$

где $\varepsilon_{2}(d ; T)=O\left(1+T p(T) r_{1}(T)\right)$ при $T \rightarrow+\infty$ равномерно по $d \in[-2,2]$,

б) для такого потенииала $q(x)$, что $T p(T) r_{1}(T) \rightarrow 0$ при $T \rightarrow+\infty$,

$$
\Delta(d ; T)=2 i \rho C_{+} C_{-} \mathcal{I}_{1}^{-1}\left(1+\varepsilon_{3}(d ; T)\right) \exp [V(T) U(T)],
$$

где $\varepsilon_{3}(d ; T)=o(1)$ при $T \rightarrow+\infty$ равномерно по $d \in[-2,2]$,

в) для такого потенциала $q(x)$, что $\operatorname{Tr}_{1}(T)$ ограничена при любом $T$,

$$
\Delta(d ; T)=2 i \rho C_{+} C_{-} \mathcal{I}_{1}^{-1}\left(1+\varepsilon_{4}(d ; T)\right) \exp [(i T / \rho) V(T)],
$$

әде $\varepsilon_{4}(d ; T)=o(1)$ при $T \rightarrow+\infty$ равномерно по $d \in[-2,2]$,

г) для такого потенииала $q(x)$, что $r_{1}(T) T \rightarrow 0$ при $T \rightarrow+\infty$,

$$
\Delta(d ; T)=2 i \rho C_{+} C_{-} \mathcal{I}_{1}^{-1}\left(1+\varepsilon_{5}(d ; T)\right),
$$

әде $\varepsilon_{5}(d ; T)=o(1)$ при $T \rightarrow+\infty$ равномерно по $d \in[-2,2]$. 
ДокАЗАТЕльСтво теоремы 2 опирается на факты, установленные в разделе 5, и проводится так же, как доказательство теоремы 2 из [2].

\section{7. ВСПОМОГАТЕЛЬНЫЕ ОЦЕНКИ В СЛУЧАЕ ДВУМЕРНОГО ИНВАРИАНТНОГО ПОДПРОСТРАНСТВА}

Необходимо отметить, что когда собственному значению $\lambda_{0}$ одноатомной задачи отвечает ИП размерности $l$, то к этому собственному значению стремятся ровно $l$ спектральных компонент периодической задачи (ср. [13]). Поэтому, ограничивая исследование спектра $\sigma(Q)$ некоторой окрестностью точки $\lambda_{0}$, которой отвечает двумерное ИП, мы вынуждены следить за двумя спектральными компонентами. Для этого введем дополнительную оценочную функцию

$$
r_{2}(T):=\sup _{t \geq T}\left(\int_{-T}^{T}|v(x, t)| e^{-2 \eta|x|} d x\right)^{1 / 2}+e^{-\eta T}, \quad T>0 .
$$

Заметим, что при таком определении $r_{2}(T)=O\left(p^{1 / 2}(T)\right)$ при $T \rightarrow+\infty$.

Для произвольного фиксированного положительного числа $C_{\kappa}$ выделяем на комплексной плоскости круг $B_{2}(T)$ радиуса $C_{\kappa} r_{2}(T)$ с центром в нуле, на котором в дальнейшем рассмотрим основное уравнение (4.2) и проведем вспомогательные оценки.

Леммы 3 и 5 в этом случае необходимо переформулировать в новых терминах.

ЛЕмма 7. Для произвольного числа $C_{\kappa}>0$ существует положительное число $T_{0}$ такое, что для любого $T \geq T_{0}$ и любого $\kappa \in B_{2}(T)$ уравнение (3.6) разречимо в $C(\mathbf{R})$. Решение $\gamma(x)=\gamma(x, \kappa)$ единственно и при $|x| \leq T$ справедливы оценки

$$
\begin{array}{lll}
|\gamma(x)| \leq C_{\gamma}\left(r_{2}(T)+p(T) e^{2 \eta|x|}\right) & u & \left|\partial_{\kappa} \gamma(x)\right| \leq C_{\gamma}^{\prime} e^{2 \eta|x|}, \\
|\ln \alpha(x)| \leq C_{\alpha}\left(r_{2}(T)|x|+p(T)\right) & u & \left|\partial_{\kappa} \ln \alpha(x)\right| \leq C_{\alpha}^{\prime}|x|,
\end{array}
$$

әде $C_{\gamma}, C_{\gamma}^{\prime}, C_{\alpha}$ и $C_{\alpha}^{\prime}$ - некоторье постояннье. Можно положить

$$
\begin{array}{lll}
C_{\gamma}=\left(1+C_{\kappa} / \eta\right)\left(C_{\varphi}+C_{\theta}\right)^{2} & u & C_{\gamma}^{\prime}=2\left(C_{\varphi}+C_{\theta}\right)^{2} / \eta \\
C_{\alpha}=C_{\varphi} C_{\theta}\left(C_{\kappa}+1\right) & u & C_{\alpha}^{\prime}=C_{\varphi} C_{\theta}+1 .
\end{array}
$$

Лемма 8. Пусть $C_{\kappa}, C_{\alpha}, C_{\alpha}^{\prime}, C_{g}$ и $C_{g}^{\prime}$ - положительные числа, причем $C_{\alpha}$ и $C_{\alpha}^{\prime}$ такие жсе, как в лемме 7, а $C_{g}$ и $C_{g}^{\prime}$ удовлетворяют условиям $C_{g}>2\left|\rho C_{+} C_{-}\right| u$ $C_{g}^{\prime}>C_{\alpha}^{\prime}\left|\rho C_{+} C_{-}\right|^{-1}$. Тогда:

а) существуют положстельные числа $T_{1}, C_{h}$ и $C_{h}^{\prime}$ такие, что для любого $T \geq T_{1}$ и любого $\kappa \in B_{2}(T)$ справедливы неравенства

$$
\begin{aligned}
|G(\kappa, T)|^{-1} & \leq C_{g} \exp \left[-2 \eta T+2 C_{\alpha} T r_{2}(T)\right], \\
\left|\partial_{\kappa} G(\kappa, T)\right| & \leq C_{g}^{\prime} T \exp \left[2 \eta T+2 C_{\alpha} T r_{2}(T)\right], \\
|H(\kappa, T)| & \leq C_{h} p(T / 2) \exp \left[2 C_{\alpha} \operatorname{Tr}_{2}(T)\right], \\
\left|\partial_{\kappa} H(\kappa, T)\right| & \leq C_{h}^{\prime}(1+T p(T / 2)) \exp \left[2 C_{\alpha} T r_{2}(T)\right]
\end{aligned}
$$


б) равномерно относительно $\kappa \in B_{2}(T)$ при $T \rightarrow+\infty$ имеет место асимптотическая формула

$$
\begin{aligned}
G^{-1}(\kappa, T)=- & 2 i \rho C_{+} C_{-}(1+O(p(T / 2))) \exp [2 i \rho T+ \\
& \left.+\kappa\left(\int_{0}^{T}-\int_{-T}^{0}\right) \theta(x) \varphi(x) d x+O\left(T p(T) r_{2}(T)\right)\right] .
\end{aligned}
$$

Как уже отмечалось, основная специфика рассматриваемой задачи связана с асимптотической малостью интеграла $\int_{-T}^{T} \theta^{2}(x) \alpha^{-2}(x) d x$. Поэтому мы должны рассмотреть асимптотическое поведение этого интеграла более подробно.

ЛЕмма 9. Пусть $\mathcal{I}_{1}=0$ u $\mathcal{I}_{2} \neq 0$. Тогда при $T \rightarrow+\infty$

$$
\int_{-T}^{T} \theta^{2}(x) \alpha^{-2}(x) d x=\mathcal{I}_{2} \kappa+o\left(r_{2}(T)\right)
$$

равномерно относительно $\kappa \in B_{2}(T)$.

ДокАЗАТЕЛЬСтво. Последовательное интегрирование по частям с учетом уравнения (3.5) приводит к следующему тождеству для произвольного $t$ :

$$
\begin{aligned}
& \int_{0}^{t} \theta^{2}(x) \alpha^{-2}(x) d x=\int_{0}^{t} \theta^{2}(x) d x+2 \kappa \int_{0}^{t} \int_{x}^{t} \theta^{2}\left(x_{1}\right) \theta(x) \varphi(x) d x_{1} d x+ \\
& \quad+2 \kappa \int_{0}^{t} \int_{x}^{t} \theta^{2}\left(x_{1}\right) \gamma(x) \theta^{2}(x) d x_{1} d x- \\
& \quad-2 \int_{0}^{t} \int_{x}^{t} \theta^{2}\left(x_{1}\right) v(x) \theta(x)(\varphi(x)+\gamma(x) \theta(x)) d x_{1} d x+ \\
& \quad+4 \int_{0}^{t} \int_{x}^{t} \int_{x_{1}}^{t} \theta^{2}\left(x_{2}\right) w\left(x_{1}\right) \theta\left(x_{1}\right)\left(\varphi\left(x_{1}\right)+\gamma\left(x_{1}\right) \theta\left(x_{1}\right)\right) \times \\
& \quad \times \alpha^{-2}(x) w(x) \theta(x)(\varphi(x)+\gamma(x) \theta(x)) d x_{2} d x_{1} d x
\end{aligned}
$$

Из равенства (7.2), учитывая что $\mathcal{I}_{1}=0$ и

$$
\int_{0}^{t} \int_{x}^{t} \theta^{2}\left(x_{1}\right) \theta(x) \varphi(x) d x_{1} d x=\int_{0}^{t} \theta^{2}\left(x_{1}\right)\left(\int_{0}^{x_{1}} \theta(x) \varphi(x) d x\right) d x_{1}
$$

мы получаем оценку

$$
\begin{gathered}
\left|\int_{-T}^{T} \theta^{2}(x) \alpha^{-2}(x) d x-2 \kappa \int_{-T}^{T} \theta^{2}(x)\left(\int_{0}^{x} \theta\left(x_{1}\right) \varphi\left(x_{1}\right) d x_{1}\right) d x\right|= \\
=O\left(r_{2}(T) p(T)\right)+O\left(r_{2}^{2}(T)\right)+o\left(r_{2}(T)\right)=o\left(r_{2}(T)\right) .
\end{gathered}
$$

Отсюда, используя соотношение

$$
\int_{-T}^{T} \theta^{2}(x)\left(\int_{0}^{x} \theta\left(x_{1}\right) \varphi\left(x_{1}\right) d x_{1}\right) d x=\int_{-\infty}^{+\infty} \theta^{2}(x)\left(\int_{0}^{x} \theta\left(x_{1}\right) \varphi\left(x_{1}\right) d x_{1}\right) d x+o(1)
$$

мы приходим к требуемой формуле. Лемма доказана.

Совершенно аналогично доказательству леммы 7 из [2] доказывается следуюшая леммa. 
ЛЕмма 10. При $T \rightarrow+\infty$

$$
\int_{-T}^{T} v(x) \theta^{2}(x) \alpha^{-2}(x) d x=\int_{-T}^{T} v(x) \theta^{2}(x) d x+O\left(p(T) r_{2}(T)\right)
$$

равномерно относительно $\kappa \in B_{2}(T)$.

\section{8. СУШЕСТВОВАНИЕ И АСИМПТОТИЧЕСКОЕ ПОВЕ ДЕНИЕ СПЕКТРАЛЬНЫХ КОМПОНЕНТ В СЛУЧАЕ ДВУМЕРНОГО ИНВАРИАНТНОГО ПОДПРОСТРАНСТВА}

Верна следующая теорема.

ТЕОрема 3. Пусть $\lambda_{0}$ - собственное значение одноатомной задачи (1.1) с двумерным ИП, т.е. $\mathcal{I}_{1}=0$ и $\mathcal{I}_{2} \neq 0$, әде $\mathcal{I}_{1}$ и $\mathcal{I}_{2}$ определень уравнениями (4.3). И пусть $C_{\kappa}$ и $d_{0}$ - положительные числа, удовлетворяюшие условиям $C_{\kappa}^{2}>$ $\left|\mathcal{I}_{2}\right|^{-1} \max \left\{C_{\theta}^{2}, 2\left|\rho C_{+} C_{-}\right| d_{0}\right\}$, если $\operatorname{Tr}_{2}(T) \rightarrow 0$ при $T \rightarrow+\infty$ и $C_{\kappa}^{2}>\left|\mathcal{I}_{2}\right|^{-1} C_{\theta}^{2}$ в противном случае. Тогда существует положстельное число $t_{0}$ такое, что для любого $T \geq t_{0}$ и любого $d \in\left[-d_{0}, d_{0}\right]$ уравнение $(4.2)$ в круге $B_{2}(T)$ имеет ровно два решения $\kappa=\kappa(d ; T)$.

ДокАЗАтЕльство. Уравнение (4.2) удобно кратко записать теперь как $\kappa^{2}=g(\kappa)$, где $g(\kappa)$ - аналитическая в круге $B_{2}(T)$ функция, зависящая также от $d, T$ и $\lambda_{0}$ :

$$
g(\kappa)=\frac{1}{\mathcal{I}_{2}}\left[\int_{-T}^{T} v \theta^{2} \alpha^{-2} d x+(H-d) G^{-1}\right]+\frac{\kappa}{\mathcal{I}_{2}}\left(\mathcal{I}_{2} \kappa-\int_{-T}^{T} \theta^{2} \alpha^{-2} d x\right) .
$$

Далее, используя результаты предыдушего раздела, оценим по модулю функцию $g(\kappa)$ на границе круга:

$$
\begin{aligned}
|g(\kappa)| \leq & {\left[\int_{-T}^{T}|v||\theta|^{2} d x(1+o(1))+\left(d_{0}+|H|\right) /|G|\right]\left|\mathcal{I}_{2}\right|^{-1}+o\left(r_{2}^{2}(T)\right) \leq } \\
\leq & \left|\mathcal{I}_{2}\right|^{-1} \int_{-T}^{T}|v||\theta|^{2} d x+o\left(r_{2}^{2}(T)\right)+\left(d_{0}+C_{h} p(T / 2) \exp \left[2 C_{\alpha} \operatorname{Tr}_{2}(T)\right]\right) \times \\
& \times 2\left|\rho C_{+} C_{-}\right|\left|\mathcal{I}_{2}\right|^{-1}(1+o(1)) \exp \left[-2 \eta T+2 C_{\alpha} T r_{2}(T)\right] .
\end{aligned}
$$

Если $\operatorname{Tr}_{2}(T) \rightarrow 0$ при $T \rightarrow+\infty$, то для больших $T$ имеем

$$
|g(\kappa)| \leq\left|\mathcal{I}_{2}\right|^{-1} \int_{-T}^{T}|v||\theta|^{2} d x+2\left|\rho C_{+} C_{-}\right|\left|\mathcal{I}_{2}\right|^{-1} d_{0} e^{-2 \eta T}+o\left(r_{2}^{2}(T)\right)<C_{\kappa}^{2} r_{2}^{2}(T) .
$$

В противном случае, если $T r_{2}(T)$ не стремится к нулю, для всех достаточно больших $T$ сушествует такое $t_{0}$, что при $T \geq t_{0}$ вьполняется неравенство $-2 \eta+4 C_{\alpha} r_{2}(T)<-\eta$. А т.к. $e^{-\eta T} T^{2} \rightarrow 0$, то $e^{-\eta T}=o\left(r_{2}^{2}(T)\right)$, и мы получаем

$$
|g(\kappa)| \leq\left|\mathcal{I}_{2}\right|^{-1} \int_{-T}^{T}|v||\theta|^{2} d x+o\left(r_{2}^{2}(T)\right) \leq\left|\mathcal{I}_{2}\right|^{-1} C_{\theta}^{2} r_{2}^{2}(T)(1+o(1))<C_{\kappa}^{2} r_{2}^{2}(T) .
$$

Следовательно, сушествует $t_{0}>0$ такое, что при $T \geq t_{0}$ на границе круга $B_{2}(T)$ верно неравенство $|g(\kappa)|<|\kappa|^{2}$. Тогда согласно теореме Руше функция $g(\kappa)-\kappa^{2}$ имеет внутри круга $B_{2}(T)$ столько же нулей, сколько функция $\kappa^{2}$, т.е. ровно два. Теорема доказана. 
В соответствии с формулой (2.3) имеем следующее следствие теоремы 3.

СЛЕДСТВИЕ 2 . Пусть $\mathcal{I}_{1}=0, \mathcal{I}_{2} \neq 0, d_{0} \geq 2, C$ - положительное число такое, что выполняется неравенство $C^{2}>\left|\mathcal{I}_{2}\right|^{-1} \max \left\{C_{\theta}^{2}, 2\left|\rho C_{+} C_{-}\right| d_{0}\right\}$. И пусть $\kappa_{1,2}(d ; T)$ при $|d| \leq d_{0}$ являются такими решениями уравнения (4.2), что $\left|\kappa_{1,2}(d ; T)\right| \leq C r_{2}(T)$ для больших $T$. Положим согласно (3.1) $\lambda_{1,2}(d ; T)=\lambda_{0}+$ $\kappa_{1,2}(d ; T)$. Тогда, для того чтобь $\lambda_{1,2}(d ; T) \in \sigma(Q)$, необходимо и достаточно, чтобы $d \in[-2,2]$. Следовательно, пара криволинейных отрезков $\left\{\lambda_{1,2}(d ; T)\right.$ : $-2 \leq d \leq 2\}$ совпадает с двумя изолированными спектральными компонентами, лежсащими вблизи $\lambda_{0}$.

Таким образом, мы доказали сушествование пары спектральных компонент вблизи $\lambda_{0}$. Приведем теперь формулы, асимптотически описывающие эти компоненты. Далее везде под выражением $z^{1 / 2}$ мы понимаем оба значения квадратного корня из комплексного числа $z$.

ТЕОрема 4. Пусть функиия $q(x) \in L^{1}(\mathbf{R})$, число $T>0$ и функиия $Q(x, T)$ определена формулой (2.1). Пусть $\lambda_{0}$ - собственное значение и $\theta(x) \in L^{2}(\mathbf{R})$ - собственная функиия задачи (1.1) такая, что $\|\theta\|_{L^{2}(R)}=1, \mathcal{I}_{1}=\int_{-\infty}^{+\infty} \theta(x)^{2} d x=0$. Пусть функиия $\varphi(x)$ - какое-либо решение уравнения (1.1) при $\lambda=\lambda_{0}$, удовлетворяющее условию $W[\theta, \varphi] \equiv 1$, причем $\mathcal{I}_{2}:=2 \int_{-\infty}^{+\infty} \theta^{2}(x)\left(\int_{0}^{x} \theta(t) \varphi(t) d t\right) d x \neq 0$. Положим $V(T):=\int_{-T}^{T}(Q(x ; T)-q(x)) \theta^{2}(x) d x, \quad U(T):=\left(\int_{0}^{T}-\int_{-T}^{0}\right) \theta(x) \varphi(x) d x . \quad$ Пусть $\rho=\sqrt{\lambda_{0}}, \quad \eta=\operatorname{Im} \rho>0$, числа $C_{\theta}$ и $C_{ \pm}$такие жее, как в (1.3) и лемме 1, а функиии $p(T)$ и $r_{2}(T)$ определены формулами (1.4) и (7.1), соответственно. Тогда, если $C^{2}>\left|\mathcal{I}_{2}\right|^{-1} \max \left\{C_{\theta}^{2}, 4\left|\rho C_{+} C_{-}\right|\right\}$и $\kappa(d ; T)$ - решение уравнения (4.2) при $d \in[-2,2]$ такое, что $|\kappa(d ; T)| \leq C r_{2}(T)$ для больших $T$, то верны следующие утвержсдения:

1) $\lambda(d ; T)=\lambda_{0}+\kappa(d ; T) \in \sigma(Q)$ npu $d \in[-2,2]$;

2) $\lambda( \pm 2 ; T)$ - края спектральных компонент;

3) имеет место асимптотическая формула для “иентров" спектральных компонент

$$
\lambda_{1,2}(0 ; T)=\lambda_{0}+s_{1}^{1 / 2}(T)
$$

әде

$$
s_{1}(T)=\left[V(T) / \mathcal{I}_{2}\right]+\varepsilon_{1}(T)
$$

$a \varepsilon_{1}(T)=o\left(r_{2}^{2}(T)\right)$ при $T \rightarrow+\infty$;

4) если при некотором положительном $\delta$ и всех достаточно больших $T$ вьполняется неравенство

$$
\left|\lambda_{1}(0 ; T)-\lambda_{2}(0 ; T)\right|<\exp ((-\eta-\delta) T)
$$

то для описания спектра вблизи $\lambda_{0}$ имеет место асимптотическая формула

$$
\lambda_{1,2}(d ; T)=\lambda_{0}+\left(2 i \rho C_{+} C_{-} d / \mathcal{I}_{2}\right)^{1 / 2} \exp [i \rho T]+\varepsilon_{2}(d ; T),
$$

где $\varepsilon_{2}(d ; T)=o\left(e^{i \rho T}\right)$ равномерно относительно $d \in[-2,2]$ при $T \rightarrow+\infty$; 
5) если при некотором положительном $\delta$ и всех достаточно больиих $T$ выполняется неравенство

$$
\left|\lambda_{1}(0 ; T)-\lambda_{2}(0 ; T)\right|>\exp ((-\eta+\delta) T)
$$

то для описания спектра вблизи $\lambda_{0}$ имеет место асимптотическая формула

$$
\lambda_{1,2}(d ; T)=\lambda_{1,2}(0 ; T)+(d / 2) s_{1}^{-1 / 2}(T) s_{2}(d ; T) e^{2 i \rho T},
$$

где для функиии $s_{2}(d ; T)$ имеют место следующие асимптотические выражсния:

а) в общем случае

$$
\ln s_{2}(d ; T)=V(T) U(T)+\varepsilon_{3}(d ; T),
$$

где $\varepsilon_{3}(d ; T)=O\left(1+T p(T) r_{2}(T)\right)$ равномерно относительно $d \in[-2,2]$ при $T \rightarrow+\infty$

б) для такого потенииала $q(x)$, что $T p(T) r_{2}(T) \rightarrow 0$ при $T \rightarrow+\infty$,

$$
s_{2}(d ; T)=2 i \rho C_{+} C_{-} \mathcal{I}_{2}^{-1}\left(1+\varepsilon_{4}(d ; T)\right) \exp [V(T) U(T)]
$$

где $\varepsilon_{4}(d ; T)=o(1)$ равномерно относительно $d \in[-2,2]$ при $T \rightarrow+\infty$,

в) для такого потенииала $q(x)$, что $\operatorname{Tr}_{2}(T)$ ограничена при любом $T$,

$$
s_{2}(d ; T)=2 i \rho C_{+} C_{-} \mathcal{I}_{2}^{-1} \exp [(i T / 2 \rho) V(T)]\left(1+\varepsilon_{5}(d ; T)\right),
$$

где $\varepsilon_{5}(d ; T)=o(1)$ равномерно относительно $d \in[-2,2]$ при $T \rightarrow+\infty$,

г) для такого потенииала $q(x)$, что $\operatorname{Tr}_{2}(T) \rightarrow 0$ при $T \rightarrow+\infty$,

$$
s_{2}(d ; T)=2 i \rho C_{+} C_{-} \mathcal{I}_{2}^{-1}\left(1+\varepsilon_{6}(d ; T)\right),
$$

где $\varepsilon_{6}(d ; T)=o(1)$ равномерно относительно $d \in[-2,2]$ при $T \rightarrow+\infty$.

ДокАЗАТЕЛЬСтво. В рассматриваемом случае уравнение (4.2) переписывается в виде

$$
\lambda_{1,2}(d ; T)=\lambda_{0}+\left[s_{1}(T)+d s(d ; T)\right]^{1 / 2},
$$

при этом $s_{1}(T)=\left\{1 / \mathcal{I}_{2}\right\}\left[\int_{-T}^{T} v \theta^{2} \alpha^{-2} d x+H G^{-1}\right](1+o(1)), s(d ; T)=\left\{-G \mathcal{I}_{2}\right\}^{-1} \times$ $(1+o(1))$, когда $T \rightarrow+\infty$. Положив здесь $d=0$, в силу лемм 8 и 10 мы получаем формулы (8.1) и (8.2). С другой стороны, чтобы разложить корень в правой части (8.5), мы должны учесть относительное асимптотическое поведение функций $s_{1}(T)$ и $s(d ; T)$ при $T \rightarrow+\infty$. Если $s_{1}(T)=o(s(d ; T))$, что гарантируется условием утверждения 4 , то формула (8.5) принимает вид $\lambda_{1,2}(d ; T)=\lambda_{0}+(d s(d ; T))^{1 / 2}+o(s(d ; T))$. С учетом леммы 8 мы приходим к результату (8.3). Если же $s(d ; T)=o\left(s_{1}(T)\right)$ при $T \rightarrow+\infty$, что выполняется при условии утверждения 5 , то (8.5) можно записать в виде (8.4), принимая $s(d ; T)=s_{2}(d ; T) e^{2 i \rho T}$. В остальном доказательство теоремы 4 проводится аналогично доказательству теоремы 2 из работы [2]. 
В заключение отметим, что с помощью техники статьи [19] аналогичные результаты могут быть получены для разностного оператора.

\section{9. ПРИМЕРЫ}

Как видно из теорем 2 и 4 , различные tb-формулы получаются при различных степенях убывания исходного потенциала одноатомной задачи. В свою очередь, степень убывания потенциала в предложенной схеме классифицируется порядком убывания функций $p(T), r_{1}(T)$ и $r_{2}(T)$. В статье [2] приведены примеры вешественных потенциалов, иллюстрируюшие различное асимптотическое поведение спектральных зон. Рассмотрение комплекснозначных потенциалов с таким же асимптотическим поведением на бесконечности, как в указанных примерах, приводит к различным степеням убывания оценочных функций $p(T)$ и $r_{1}(T)$, а следовательно, к различным tb-формулам в случае одномерного ИП.

Новым моментом, качественно отличаюшим случай комплекснозначного потенциала от вещественного, является возможность возникновения присоединенных функций. Именно это мы показываем в приведенных ниже примерах. Достаточно рассмотреть простейший потенциал, состояший из двух дельта-функций.

Для потенциала с одной дельта-функцией $q(x)=q_{0} \delta(x), q_{0} \in \mathbf{C}$, при условии $\operatorname{Re} q_{0}<0$ сушествует лишь одно собственное значение $\lambda_{0}=-q_{0}^{2} / 4$ с одномерным ИП. Если рассмотреть потенциал, представляюший собой сумму двух дельта-функций, т.е. $q(x)=q_{0}(\delta(x-a)+\delta(x+a)), a>0, q_{0} \in \mathbf{C} \backslash\{0\}$, то для определенных значений $q_{0}$ мы получим пример сушествования собственного значения с двумерным ИП. Однако трехмерного ИП ни при каких $q_{0}$ не возникает. Простейший пример потенциала с трехмерным ИП может быть построен в виде суммы двух дельта-функций с разными весами: $q(x)=q_{1} \delta(x-a)+q_{2} \delta(x+a), a>0, q_{1}, q_{2} \in \mathbf{C} \backslash\{0\}$. Отметим, что потенциал, состояший из суммы двух дельта-функций, традиционно рассматривается как модель водородоподобных атомов и двухатомных молекул $[20,21]$. Уравнение, описываюшее связанные состояния в этой модели, сохраняет свой вид как для вешественных, так и для комплексных параметров $q_{1}$ и $q_{2}$ :

$$
q_{1} q_{2} e^{4 i \rho a}=\left(q_{1}-2 i \rho\right)\left(q_{2}-2 i \rho\right) .
$$

Решение этого трансцендентного уравнения в алгебраической форме затруднительно. Тем не менее, как отмеченно в [21], в случае одинаковых весов корни уравнения, а следовательно, и собственные значения, явно выражаются через функцию Ламберта $W(z)$ (по определению $W(z) \exp (W(z)) \equiv z)[22]$.

Рассмотрим теперь соответствующие периодические задачи. Поскольку все эти задачи явно решаемы, то tb-формулы в данном случае могут быть получены двумя способами: из теорем 2 или 4 и прямым асимптотическим исследованием при $T \rightarrow \infty$ конкретной функции Ляпунова. Приведем результаты вычислений, проведенных с помошью компьютерной алгебраической системы Maple $V$ [23]. 
Рассмотрим потенциал типа $q(x)=q_{1} \delta(x-a)+q_{2} \delta(x+a)$. Фиксируем произвольное число $\lambda_{0} \in \mathbf{C} \backslash \mathbf{R}_{+}$и положим $\lambda_{0}=\rho^{2}, \eta:=\operatorname{Im} \rho>0$. Подставляя $\lambda_{0}$ в уравнение (9.1), находим связь между $q_{1}$ и $q_{2}$. Далее, для любого $q_{1} \neq 2 i \rho$ однозначно определяется $q_{2}$ и для этой пары весов выбранное число $\lambda_{0}$ будет являться собственным значением уравнения (1.1). Если числу $\lambda_{0}$ соответствует одномерное ИП, то по теореме 2 мы получаем следуюшую tb-формулу:

$$
\lambda(d ; T)=\rho^{2}-\frac{4 i d \rho^{3}}{2 a A B+A+B} e^{2 i T \rho}+o\left(e^{-2 T \eta}\right), \quad-2 \leq d \leq 2 .
$$

Здесь и далее $A=q_{1}-2 i \rho, B=q_{2}-2 i \rho$. Если исследовать асимптотическое поведение функции Ляпунова для данного $\lambda_{0}$, то естественным образом возникает знаменатель формулы (9.2). Вследствие теоремы 2 обрашение в нуль знаменателя формулы (9.2) дает условие сушествования по крайней мере одной присоединенной функции, отвечаюшей выбранному собственному значению $\lambda_{0}$. Таким образом, в соответствии с теоремой 4 , если $\rho$ является простым корнем знаменателя (9.2), а следовательно, кратным корнем уравнения (9.1), имеет место следуюшая tb-асимптотическая формула:

$$
\lambda_{1,2}(d ; T)=\rho^{2}+2 i \rho^{2}\left(\frac{2 d A B}{A^{2}+B^{2}}\right)^{1 / 2} e^{i T \rho}+o\left(e^{-T \eta}\right), \quad-2 \leq d \leq 2 .
$$

Эта же формула получается прямым вычислением. Аналогично нули знаменателя формулы (9.3), т.е. нули кратности три уравнения (9.1), соответствуют собственному значению с ИП размерности три (например, при $a=1$ можно взять $\rho=0.67715+i 0.17858$, $\left.q_{1}=2 i \rho+(i-1) / 2, q_{2}=2 i \rho-(i+1) / 2\right)$.

Далее проанализируем геометрию спектральных компонент, определяемую главными членами tb-асимптотических формул (9.2), (9.3). Прежде всего отметим симметрию возникающих картин относительно $\lambda_{0}=\rho^{2}$. Эти картины при каждом $T$ строятся из отрезков, количество которых равно размерности соответствуюшего ИП. Единственной точкой пересечения этих отрезков является $\lambda_{0}$, а все углы между ними равны. Каждая из картин врашается вокруг $\lambda_{0}$, и угол поворота линеен по $T$. Однако скорости врашения различны. Длины отрезков убывают экспоненциально. Возникновение всякой новой присоединенной функции степенным образом увеличивает диаметр tb-спектра.

При $q_{1}=q_{2}=q_{0}$ уравнение (9.1), определяюшее собственные значения, распадается на два независимых уравнения, отвечаюших четной и нечетной собственным функциям, а именно

$$
u e^{u}= \pm q_{0} a e^{q_{0} a},
$$

где $u=\left(q_{0}-2 i \rho\right) a$. Решения этих уравнений записываются через функцию Ламберта $W$ следуюшим образом:

$$
u=W\left( \pm q_{0} a e^{q_{0} a}\right) .
$$

Заметим, что соотношение (9.4) для любого $q_{0}$ определяет бесконечное число решений в силу многолистности функции $W$. Однако интересуюшие нас решения определяются 
дополнительным условием $\operatorname{Im} \rho>0$, что накладывает ограничения на $q_{0}$ для сушествования собственного значения. Например, при вешественных $q_{0}>0$ собственных значений нет. Подробньй анализ показывает, что для любого $q_{0}$ число собственных значений всегда конечно, хотя и может быть сколь угодно большим при правильном выборе $q_{0}$. Аналогичным образом разрешаются условия двумерности ИП:

$$
\rho=\frac{-i}{2 a}\left(1+W\left(\mp \frac{1}{e}\right)\right), \quad \operatorname{Im} \rho>0 .
$$

Далее надо обеспечить выполнение условия (9.1) подбором соответствующего значения $q_{0}=q_{1}=q_{2}$.

И, наконец, для "гребенки Дирака" ( $\left.a=0, q_{1}+q_{2}=q_{0}, \rho=-i q_{0} / 2\right)$ приведем уточненную tb-формулу:

$$
\lambda(d ; T)=\rho^{2}+2 d \rho^{2} e^{2 i T \rho}+4 i d^{2} T \rho^{3} e^{-4 T \eta}+O\left(e^{4 i T \rho}\right), \quad-2 \leq d \leq 2 .
$$

Третье слагаемое в этой формуле содержит предэкспоненциальный множитель $T$. Ранее мы наблюдали появление предэкспоненты во втором слагаемом для медленно убываюших потенциалов. Формула (9.5) показывает, что подобный множитель появляется в третьем слагаемом даже для потенциалов нулевого радиуса.

Авторы выражают искреннюю благодарность Б. С. Павлову и М. М. Фаддееву за полезные обсуждения излагаемых вопросов, а также рецензенту за содержательные замечания. На различных этапах работы над данной статьей исследования были поддержаны Фондом помощи американского математического обшества странам бывшего Советского Союза, Международной Соросовской программой образования в области точных наук и грантом INTAS-93-1813.

\section{Список литературы}

[1] V. L. Oleinik. J. Stat. Phys. 1990. V. 59. № 3/4. P. 665-678.

[2] А. Л. Миронов, В. Л. Олейник. ТМФ. 1994. Т. 99. № 1. С. 103-120.

[3] И. М. Глазман. Прямые методы качественного спектрального анализа сингулярных дифференциальных операторов. М.: Физматгиз, 1963.

[4] М. А. Наймарк. Линейные дифференциальные операторы. М.: Наука, 1969.

[5] Э. Ч. Титчмари. Разложения по собственным функциям, связанные с дифференциальными уравнениями второго порядка. Т. 2. М.: ИИЛ, 1961.

[6] М. Рид, Б. Саймон. Методы современной математической физики. Анализ операторов. Т. 4. М.: Мир, 1982.

[7] М. И. Серов. ДАН СССР. 1960. Т. 131. № 1. С. 27-29.

[8] Ф. С. Рофе-Бекетов. ДАН СССР. 1963. Т. 152. № 6. С. 1312-1315.

[9] В. А. Ткаченко. ДАН СССР. 1964. Т. 155. № 2. С. 289-291.

[10] А. И. Ансельм. Введение в теорию полупроводников. М.: Наука, 1978.

[11] Ф. Бассани, Дж. Пастори Парравичини. Электронные состояния и оптические переходы в твердых телах. М.: Наука, 1982. 
[12] Дж. Каллуәй. Теория энергетической зонной структуры. М.: Мир, 1969.

[13] G. V. Galloonov, V. L. Oleinik, B. S. Pavlov. J. Math. Phys. 1993. V. 34. № 3. P. 936.

[14] Л. А. Пастур, В. А. Ткаченко. Мат. заметки. 1991. Т. 50. № 4. С. 88-95.

[15] V. A. Tkachenko. Ann. Math. 1996. V. 143. P. 181.

[16] V. A. Tkachenko. Adv. Sov. Math. 1994. V. 19. P. 41.

[17] В. Э. Лянце. Несамосопряженный дифференциальный оператор второго порядка на полуоси. Добавление к кн. М. А. Наймарк. Линейные дифференциальные операторы. М.: Наука, 1969.

[18] З. Флюгге. Задачи по квантовой механике. Т. 1. М.: Мир, 1974.

[19] A. L. Mironov, V. L. Oleinik. J. Stat. Phys. 1994. V. 75. № 1/2. P. 317.

[20] A. A. Frost. J. Chem. Phys. 1956. V. 25. № 6. P. 1150.

[21] T. C. Scott, J.F. Babb, A. Dalgarno, J.D. Morgan III. J. Chem. Phys. 1993. V. 99. № 4. P. 2841.

[22] R. M. Corless, G. H. Gonnet, D. E. G. Hare, D. J. Jeffrey, D. E. Knuth. Adv. Comput. Math. (in press).

[23] B. W. Char, K. O. Geddes, G.H. Gonnet, B. L. Leong, M. B. Monagan, S. M. Watt. Maple V Language Reference Manual. New York: Springer, 1991.

Поступила в редакцию 26.II.1997 г.

\section{A.L. Mironov, V.L. Oleinik \\ LIMITS OF APPLICABILITY \\ OF THE TIGHT BINDING APPROXIMATION FOR COMPLEX-VALUED POTENTIAL FUNCTION}

We consider a one-dimensional Schrödinger operator with periodic potential that is constructed as a sum of shifts of a given complex-valued potential $q \in L^{1}(\mathbf{R})$. A mathematical basis of the tight binding approximation in this case is given. Let $\lambda_{0}$ be an isolated eigenvalue of Schrödinger operator with potential $q$. Then for the operator with periodic potential there exists a continuos spectrum that lies near $\lambda_{0}$. An asymptotic behavior of this part of the spectrum for the cases of one- and two-dimensional invariant subspace corresponding to $\lambda_{0}$ when the period tends to infinity is studied. 